\title{
Inter- and intra-specific gene-density-correlated radial chromosome territory arrangements are conserved in Old World monkeys
}

\author{
H. Tanabe, ${ }^{a}$ K. Küpper, ${ }^{b}$ T. Ishida, ${ }^{c}$ M. Neusser ${ }^{b}$ and H. Mizusawa ${ }^{a}$ \\ a Cell Bank Laboratory, Division of Genetics and Mutagenesis, National Institute of Health Sciences, Tokyo (Japan); \\ ${ }^{b}$ Department of Biology II, Ludwig-Maximilians University, Munich (Germany); \\ ${ }^{c}$ Unit of Human Biology and Genetics, Department of Biological Sciences, Graduate School of Science, The University of Tokyo, \\ Tokyo (Japan)
}

\begin{abstract}
Recently it has been shown that the gene-density correlated radial distribution of human 18 and 19 homologous chromosome territories (CTs) is conserved in higher primates in spite of chromosomal rearrangements that occurred during evolution. However, these observations were limited to apes and New World monkey species. In order to provide further evidence for the evolutionary conservation of gene-density-correlated CT arrangements, we extended our previous study to Old World monkeys. They comprise the remaining species group to be analyzed in order to obtain a comprehensive overview of the nuclear topology of human 18 and 19 homologous CTs in higher primates. In the present study we investigated four lymphoblastoid cell lines from three species of Old World monkeys by three-dimensional fluorescence in situ hybridization (3D-FISH): two individuals of Japanese macaque (Macaca fuscata), crab-eating macaque (Macaca fascicularis), and an
\end{abstract}

interspecies hybrid individual between African green monkey (Cercopithecus aethiops) and Patas monkey (Erythrocebus patas). Our data demonstrate that gene-poor human 18 homologous CTs are located preferentially close to the nuclear periphery, whereas gene-dense human 19 homologous CTs are oriented towards the nuclear center in all cell lines analyzed. The gene-density-correlated positioning of human 18 and 19 homologous CTs is evolutionarily conserved throughout all major higher primate lineages, despite chromosomal inversions, fusions, fissions or reciprocal translocations that occurred in the course of evolution in these species. This remarkable preservation of a gene-density-correlated chromatin arrangement gives further support for a functionally relevant higher-order chromatin architecture.

Copyright $\odot 2005$ S. Karger AG, Basel
Chromosome territories (CTs) in the interphase nucleus are not randomly dispersed but highly organized in animal and plant cells (for review see Lamond and Earnshaw, 1998; Chevret et al., 2000; Leitch, 2000; Cremer and Cremer, 2001; Parada and Misteli, 2002). However, the underlying molecular

\footnotetext{
Supported by the Health Sciences Research Grants, Ministry of Health, Labor and Welfare (Japan) and by the grant of the Japan Health Sciences Foundation.

Received 20 October 2003; revision accepted 17 November 2003.

Request reprints from: Hideyuki Tanabe, Cell Bank Laboratory Division of Genetics and Mutagenesis, National Institute of Health Sciences 1-18-1, Kamiyoga, Setagaya-ku, Tokyo 158-8501 (Japan) telephone: +81-3-3700-9874; fax: +81-3-3707-6950; e-mail: tanabe@nihs.go.jp

Present address of Hideyuki Tanabe: Department of Biosystems Science School of Advanced Sciences, The Graduate University for Advanced Studies (Sokendai), Shonan Village, Hayama, Kanagawa 240-0193 (Japan) telephone: +81-46-858-1573; fax: +81-46-858-1544 e-mail: tanabe-hideyuki@soken.ac.jp
}

mechanisms for the establishment and maintenance of higher order chromatin arrangements are still largely unknown. Several recent studies shed some light on certain aspects that might play a role in the spatial organization of CTs during the cell cycle, in different cell types, during cell differentiation or after malignant transformation (Bridger et al., 2000; Cremer et al., 2001, 2003; Bickmore et al., 2002; Kozubek et al., 2002). In addition, Habermann et al. (2001) and Tanabe et al. (2002a, b) studied the radial organization of CTs in birds and non-human primates.

For human lymphocytes and lymphoblastoid cells, but also in certain tumors, it has been demonstrated that the radial positioning of CTs is highly correlated with gene density (Croft et al., 1999; Boyle et al., 2001; Cremer et al., 2001, 2003). Striking evidence of this phenomenon has been brought on by the different radial positioning of human 18 and 19 CTs which are of similar size (77 and $63 \mathrm{Mbp}$, respectively) but differ strongly in their gene densities (8.7 and 27.9 genes/Mbp, respectively) \begin{tabular}{lll}
\hline KARGER & $\begin{array}{l}\text { Fax }+41613061234 \\
\text { E-mail karger@karger.ch } \\
\text { www. }\end{array}$ & ( 2005 S. Karger AG, Basel \\
0301-0171/05/1083-0255 $\$ 22.00 / 0$
\end{tabular}
Accessible online at:

www. karger.com/cgr 


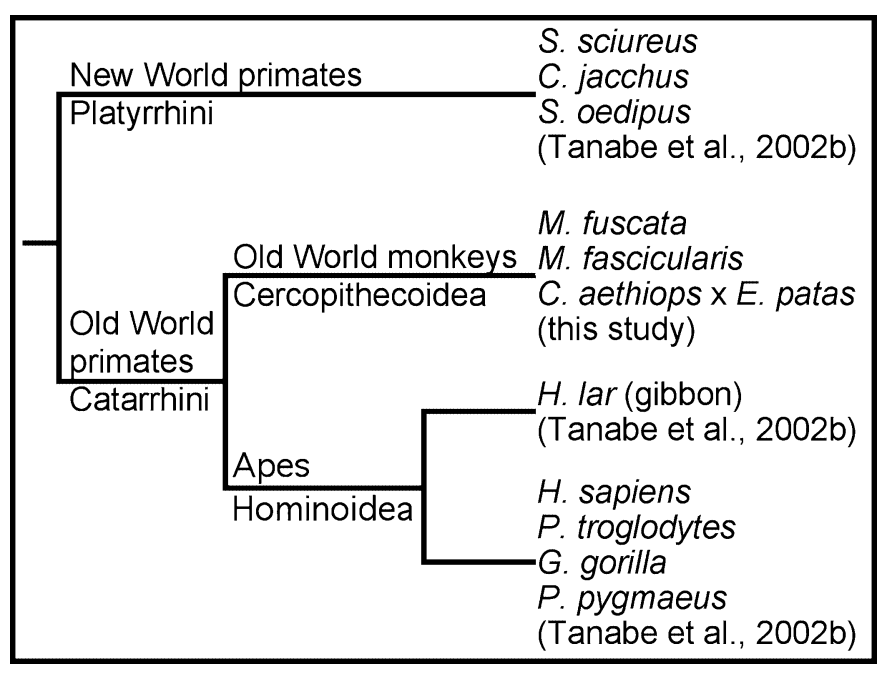

Fig. 1. Phylogenetic relationship of higher primate species for which the radial arrangement of human 18 and 19 homologous chromosome territories was assessed in S-phase nuclei of lymphoblastoid cells.

(http://www.ncbi.nlm.nih.gov/genome/guide/human/). The radial positioning of gene poor human $18 \mathrm{CTs}$ was noticed to be close to the nuclear periphery, whereas gene dense human 19 CTs were preferentially located near the nuclear center. The topology of human 18 and 19 homologous CTs has been previously examined in higher primate species showing that genedensity correlated radial arrangements of human 18 and 19 homologous CTs were highly conserved during primate evolution irrespective of multiple chromosomal rearrangements that occurred in some of these primate species, like in gibbons (Tanabe et al., 2002b). However, this study was confined to apes and New World monkey species. In order to obtain a comprehensive overview on radial chromatin arrangements of human 18 and 19 homologous CTs in all major taxonomic groups of higher primates, we extended our previous study (Tanabe et al., 2002b) to Old World monkey species (Fig. 1). Old World monkeys are classified to the infraorder Catarrhini, which diverged from the Platyrrhini (New World monkeys) about 40 million years ago. Subsequent branching of Catarrhini into Cercopithecoidea (Old World monkeys) and Hominoidea (human and apes) occurred about 25-30 million years ago (Goodman et al., 1994).

In this study we have investigated the radial positioning of human 18 and 19 homologous CTs by 3D-FISH in cell nuclei from lymphoblastoid cell lines of Old World monkey species, represented by two individuals of Japanese macaque (Macaca fuscata), one individual of crab-eating macaque (Macaca fascicularis), and a guenon interspecies hybrid individual. The latter was the offspring of a cross between African green monkey (Cercopithecus aethiops) and Patas monkey (Erythrocebus patas). Macaques have retained a very conservative karyotype with $2 n=42$, which appears to be essentially the same in all species. Overall, a high degree of syntenic conservation between human and macaque chromosomes has been maintained for more than 20 million years, including human 18 and 19 homologous chromosomes (Stanyon et al., 1983, 1990; Wienberg et al., 1992; Wienberg and Stanyon, 1998). Guenon karyotypes were evolutionary derived from those of macaques by a series of fissions (Finelli et al., 1999), which, however, did not involve human 18 and 19 homologs in the species analyzed to date by chromosome painting. Our present data provide further evidence for an evolutionary conservation of radial chromatin arrangements that are correlated to gene density in representatives of all higher primate lineages.

\section{Materials and methods}

\section{Cell lines and slide preparation}

Lymphoblastoid cell lines were established from two individuals of Japanese macaque (Macaca fuscata, JAM-H21 and JAM-T46) and one individual of crab-eating macaque (Macaca fascicularis, TK-246) by spontaneous transformation. An Epstein-Barr virus-transformed lymphoblastoid cell line, Mitas, was established from an African green monkey (Cercopithecus aethiops) $\times$ Patas monkey (Erythrocebus patas) interspecies hybrid individual. The peripheral blood samples from these individuals were provided by the Primate Research Institute, Kyoto University (Ishida, 1991). All cell lines were karyotypically normal, with a diploid number of 42 chromosomes in cell lines JAM-H21, JAM-T46, and TK-246 and $2 n=57$ in the normal diploid cell line Mitas, which showed an intermediate chromosome number of the parental species $(2 n=60$ in the African green monkey and $2 n=54$ in the Patas monkey). In order to obtain three-dimensionally (3D) preserved cell nuclei, cells were cultured and fixed according to Solovei et al. (2002). For S-phase detection, cells were pulse-labeled by 5-bromo-deoxyuridine (BrdU) for $1 \mathrm{~h}$ prior to fixation.

Probe preparation, three-dimensional fluorescence in situ hybridization (3D-FISH) and fluorescence detection

For the delineation of human 18 and 19 homologous chromosomes in Old World monkey species, we used human 18 and 19 homologous painting probes from gorilla and orangutan, respectively, established by flow-sorted chromosomes as described elsewhere (Müller et al., 1997). Probe labeling was performed by DOP-PCR (Telenius et al., 1992) in the presence of Biotin16-dUTP or Digoxigenin-11-dUTP (Roche). 3D-FISH, detection of labeled probes, incorporated $\mathrm{BrdU}$, and DNA counterstaining were performed according to the protocols described elsewhere (Cremer et al., 2001; Solovei et al., 2002).

\section{Confocal microscopy and quantitative evaluation of the nuclear} positioning of painted CTs by assessment of the $3 D$ relative radial distribution (3D-RRD)

Nuclei were scanned with an axial distance of $100 \mathrm{~nm}$ or $200 \mathrm{~nm}$ using a confocal laser scanning microscope (LSM 510 META, Carl Zeiss Co., Ltd.) equipped with a $63 \times / 1.4$ Plan-Apochromat objective. For each optical section, images were recorded sequentially for all three fluorochromes (FITC, $\mathrm{Cy} 3$, and Cy5). 24-bit color confocal image stacks with a pixel size of $66 \mathrm{~nm}$ were converted to 8-bit gray scale image stacks with $256 \times 256$ pixels in each channel for subsequent quantitative evaluation. The image stacks were processed with ImageJ1.30 software (http://rsb.info.nih.gov/ij/). 3D reconstructions of hybridized nuclear image stacks were obtained by Amira 3.0 TGS (http://www.amiravis.com/) software.

A detailed description of the quantitative 3D evaluation of light optical serial sections by a voxel (volume element)-based algorithm is published elsewhere (Cremer et al., 2001, 2003). In brief, as a first step, the geometrical center and the border of the nucleus were determined by using the 3D data set of the DNA counterstain. The distance between the nuclear center and each point located on the segmented nuclear border was given as the relative radius $\left(r_{0}=100\right)$. The segmented nuclear space was divided into 25 equidistant shells. In a second step, segmentation of CTs was performed in each 3D stack representing the color channels for painted CTs. For each voxel located within the segmented nuclear border, the relative distance $\mathrm{r}$ from the nuclear center was calculated as a fraction of $\mathrm{r}_{0}$. For each shell, all voxels assigned to a 

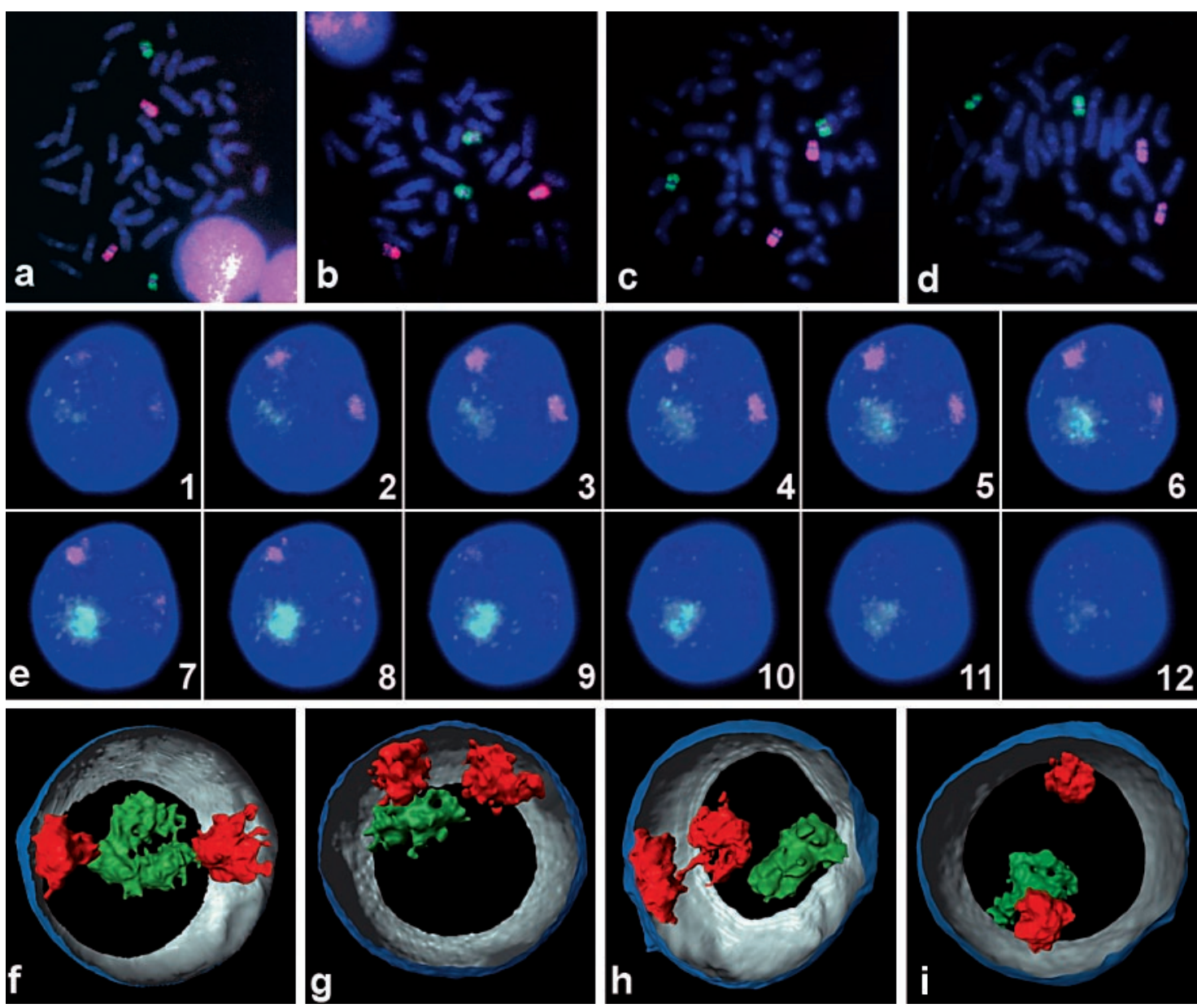

Fig. 2. Visualization of human 18 and 19 homologous chromosomes and chromosome territories in Old World monkey lymphoblastoid cells. (a-d) Metaphase analyses were carried out in each Old World monkey species on the metaphase spreads prepared from (a) JAM-H21, (b) JAM-T46, (c) Mitas, and (d) TK-246 cell lines. All these species have a single separate pair of painted homologous chromosomes corresponding to both human chromosomes 18 and 19. (e) A partial gallery of 100-nm serial light optical sections with every third section is shown from 3D-FISH scanned nuclei of Mitas cell line. Human chromosome 18 and 19 paints are shown in red and green, respectively, and nuclear DNA counterstain with TOPRO-3 is shown in blue. (f-i) 3D-reconstructed human 18 and 19 homologous chromosome territories with the partially reconstructed nuclear border (outside: blue; inside: silver-gray) are shown in (f) JAM-H21, (g) JAM-T46, (h) Mitas, and (i) TK-246 cell nuclei. Human 18 and 19 homologous chromosome territories are colored in red and green, respectively.

given $\mathrm{CT}$ were identified and the fluorescence intensities derived from each color channel were summed up. This procedure yielded the individual relative DNA content within each shell for painted CTs as well as the overall DNA content reflected by the DNA counterstain. The sum of the voxel intensities measured in each nucleus was set to $100 \%$ for each fluorochrome. As a final step, using this normalization, the relative DNA content within a nuclear shell as a function of the relative distance $r$ from the 3D center in the entire set of evaluated nuclei was plotted as a graph.

To test for significant differences $(P=0.05)$ in the distribution curves for each fluorochrome, two different tests were applied: (1) the Mann-Whitney $\mathrm{U}$ test, which measures the distribution differences of two independent samples in a nonparametric mode, and (2) the more stringent two-sample Kolmogoroff-Smirnoff test were applied to test for significant differences in the cumulative frequencies of voxel intensities for each fluorochrome. For a comparison of the distribution of human 18 and 19 homologous CTs between species, the average relative radius of the voxel distribution of human 18 and 19 homologous chromosome materials (referred to as average $\%$ in Tables 1 and 2) was calculated from all evaluated nuclei. For the deter- mination of the number of nuclei with a conserved radial arrangement showing that human 18 homologous CTs were located in a more peripheral location than human 19 homologous CTs, the number of individual nuclei showing an "inversed pattern" (CT 18 material was located more centrally compared with CT 19 material) was determined by measuring the average radial value of voxels assigned to a CT within a single nucleus.

\section{Results}

Hybridization to metaphases confirmed that all four cell lines used in our study have one separate pair of homologs corresponding to human 18 and 19 chromosomes (Fig. 2a-d) as previously reported for macaque and guenon species (Wienberg et al., 1992; Stanyon et al., 1995; Finelli et al., 1999). In 3D 


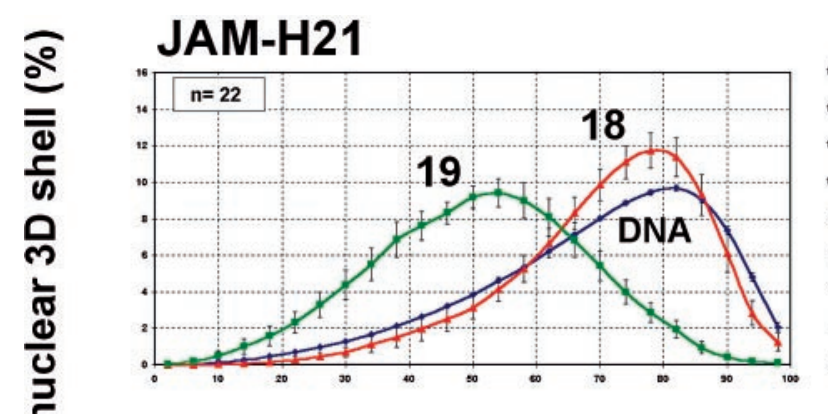

\section{Mitas}

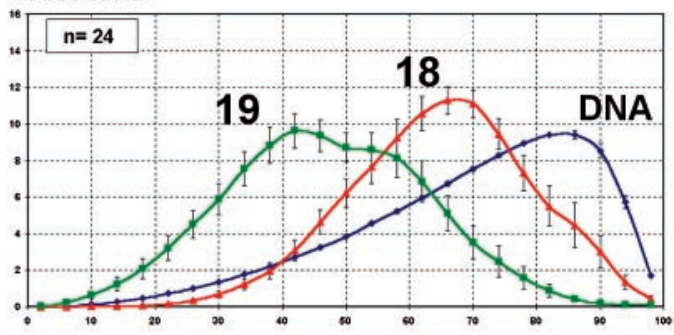

JAM-T46

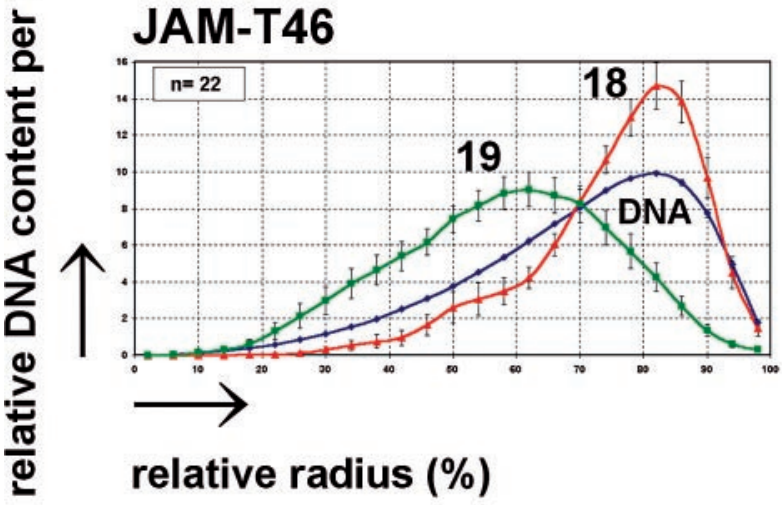

TK-246

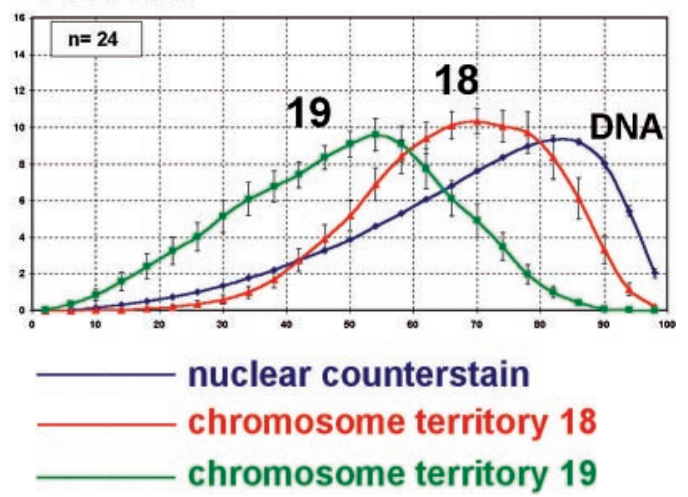

Fig. 3. Quantitative 3D evaluation of radial arrangements of human 18 and 19 homologous chromosome territories in Old World monkey cell nuclei. The name of the cell lines is indicated on the upper left side of each graph. The abscissa denotes the relative radius of the nuclear shells (center $=0$; periphery $=100$ ), the ordinate denotes the normalized sum of the voxel intensities for a respective fluorochrome belonging to a given shell. For normalization, the area under the curve for each color (total relative DNA content) was set to 100 . N equals the number of 3D evaluated nuclei. Blue curves represent counterstained DNA. All graphs indicate that human 18 homologous chromosome territories are consistently distributed close to the nuclear border, while human 19 homologous chromosome territories are located in the nuclear interior. Vertical bars indicate standard deviations of the mean for each shell.

preserved cell nuclei from all Old World monkey individuals investigated here, human 18 and 19 homologous CTs were localized at distinct radial $3 \mathrm{D}$ positions within the nucleus: human 18 homologous CTs were preferentially orientated towards the nuclear periphery, whereas human 19 homologous CTs were located preferentially near the nuclear interior. A partial gallery of 100-nm serial light optical sections (every third section shown) from a representative 3D-scanned nucleus of the Mitas cell line derived from the interspecies hybrid individual of African green monkey $\times$ Patas monkey is depicted in Fig. 2e. Representative 3D reconstructed images of CTs and the nuclear border from each cell line are illustrated in Fig. $2 \mathrm{f}-\mathrm{i}$.

For quantitative 3D evaluation of radial arrangements of human 18 and 19 homologous CTs, a set of 22-24 nuclei per cell line was evaluated as described previously (Cremer et al., 2001; Tanabe et al., 2002b). The resulting data are graphically illustrated in Fig. 3. From all the four Old World monkey cell lines the distribution curves of human 18 homologous CTs were displaying peaks of maximum DNA content between 66 and $82 \%$ of the relative radius, whereas human 19 homologous CTs were showing peaks of maximum DNA content between 42 and $62 \%$ of the relative radius (Fig. 3; Table 1). For each experiment, the distribution curves from human 18 and 19 homologous CTs were significantly different at $P=0.001$, as determined by both the Mann-Whitney U test and the Kolmogoroff-Smirnoff test.

For a more comprehensive comparison, we calculated the average relative radii, the distances between peaks of maximum DNA content, and the distances between average relative radii of human 18 and 19 homologous CTs (Table 1 ). The results indicate that in all four Old World monkey cell lines the average relative radii were similar to the peaks of maximum DNA content, and both the distances between peaks and between averages were in the same range suggesting that radial arrangements of human 18 and 19 homologous CTs were essentially conserved in all the cell lines of Old World monkeys analyzed in this study.

\section{Discussion}

The Japanese and the crab-eating macaques as well as an interspecies hybrid between African green monkey and Patas monkey have been included in the present study. In combination with the primate species previously analyzed by Tanabe et 
The two individuals of Japanese macaque analyzed also showed a very similar distribution pattern of human 18 and 19 homologous CTs, when compared with each other (Fig. 3). Although we could not study a larger number of individuals, the results indicate that this radial chromatin organization only shows limited variability in different individuals of a nonhuman primate species. These findings correlate well with those in different human lymphoblastoid cell lines (Boyle et al., 2001; Cremer et al., 2001). In addition, investigation of the interspecies hybrid individual (Mitas cell line) enabled us to explore whether these arrangements are altered or still conserved even in cases when genomes of different species are mixed during meiosis. Again, our results showed clearly distinct patterns of radial CT arrangement with the same orientation of chromosome 18 and 19 homologous CTs as in all other primate species investigated to date.

For better comparison of radial CT arrangements in different primate lineages, we summarized the calculations of data from the previous results reported in Tanabe et al. (2002b), including human, apes, and three New World monkey species (Table 2), and those from Old World monkey species, analyzed here (Table 1). The averages of peaks of maximum DNA content and the average relative radii of human 18 and 19 homologous CTs were quite similar in all three major primate groups: human and apes, Old World monkeys, and New World monkeys (average of peaks of maximum DNA content for human 18 homologous CTs: $74.0-84.8 \%$ and for human 19 homologous CTs: $49.0-53.6 \%$, see Tables 1 and 2). In addition, the distances between average relative radii of human 18 and 19 homologous CTs were quite similar in all three major groups, ranging from 18.4 to $24.5 \%$, although the average distances between peaks of maximum DNA content in Old World monkeys were slightly lower (21.0\%) as compared with human, apes $(31.2 \%)$ and New World monkeys (33.3\%). Furthermore, fre- quencies of nuclei where human 18 homologous CTs show a more peripheral location than human 19 homologous CTs were consistently over $90 \%$ in all the primate lineages. These could be an important aspect related to the stability of the radial CT positioning in normal cell types as compared with human tumor cell lines where more variable frequencies ranging from 69.0 to $91.3 \%$ (Cremer et al., 2003) were observed.

By conclusion, the topological organization of the radial 3Dpositioning of both human 18 and 19 homologous CTs from human to New World monkeys has been highly conserved in the course of 40 million years of evolution. The data indicated a striking conservation of radial arrangements of human 18 and 19 homologous CTs, despite numerous chromosomal rearrangements that occurred during the primate evolution. Neither inversions observed in great apes, multiple reciprocal translocations in gibbons nor fissions in guenons seemed to have a drastic effect on the gene-density-correlated radial organization of chromatin. This evolutionary conservation of gene-densitycorrelated radial chromatin arrangements in different primate species gives indirect but strong support for a possible functional role of higher order chromatin architecture. Further studies on the radial CT $3 \mathrm{D}$ positioning by both $3 \mathrm{D}$ and $4 \mathrm{D}$ approaches using live cells (Phair and Misteli, 2000; Gerlich et al., 2001; Kimura and Cook, 2001; Haraguchi et al., 2002; Walter et al., 2003) would enable us to understand the higher order nuclear architecture in more detail.

\section{Acknowledgements}

The authors are grateful to Ms. Kayoko Suenaga, Carl Zeiss Co. Ltd., for her helpful assistance and facilities for operating Zeiss LSM 510 META confocal microscope and to Ms. Maki Hojo, JCRB Cell Bank, for her works of metaphase analyses. The authors are also grateful to Dr. Stefan Müller, LMU, Munich, for critical reading of the manuscript.

\section{References}

Bickmore WA, Teague P: Influences of chromosome size, gene density and nuclear position on the frequency of constitutional translocations in the human population. Chromosome Res 10:707-715 (2002).

Boyle S, Gilchrist S, Bridger JM, Mahy NL, Ellis JA Bickmore WA: The spatial organization of human chromosomes within the nuclei of normal and emerin-mutant cells. Hum Mol Genet 10:211-219 (2001).

Bridger JM, Boyle S, Kill IR, Bickmore WA: Re-modelling of nuclear architecture in quiescent and senescent human fibroblasts. Curr Biol 10:149-152 (2000).

Chevret E, Volpi EV, Sheer D: Mini review: form and function in the human interphase chromosome Cytogenet Cell Genet 90:13-21 (2000).

Cremer M, von Hase J, Volm T, Brero A, Kreth G, Walter J, Fischer C, Solovei I, Cremer C, Cremer $\mathrm{T}$ : Non-random radial higher-order chromatin arrangements in nuclei of diploid human cells. Chromosome Res 9:541-567 (2001).

Cremer M, Küpper K, Wagler B, Wizelman L, von Hase J, Weiland Y, Kreja L, Diebold J, Speicher MR, Cremer T: Inheritance of gene density-related higher order chromatin arrangements in normal and tumor cell nuclei. J Cell Biol 162:809-820 (2003).
Cremer T, Cremer C: Chromosome territories, nuclear architecture and gene regulation in mammalian cells. Nat Rev Genet 2:292-301 (2001).

Croft JA, Bridger JM, Boyle S, Perry P, Teague P, Bickmore WA: Differences in the localization and morphology of chromosomes in the human nucleus. J Cell Biol 145:1119-1131 (1999).

Finelli P, Stanyon R, Plesker R, Ferguson-Smith MA, O'Brien PC, Wienberg J: Reciprocal chromosome painting shows that the great difference in diploid number between human and African green monkey is mostly due to non-Robertsonian fissions. Mamm Genome 10:713-718 (1999).

Gerlich D, Beaudouin J, Gebhard M, Ellenberg J, Eils R: Four-dimensional imaging and quantitative reconstruction to analyse complex spatiotemporal processes in live cells. Nat Cell Biol 3:852-855 (2001)

Goodman M, Bailey WJ, Hayasaka K, Stanhope MJ, Slightom J, Czelusniak J: Molecular evidence on primate phylogeny from DNA sequences. Am J Phys Anthropol 94:3-24 (1994).
Habermann FA, Cremer M, Walter J, Kreth G, von Hase J, Bauer K, Wienberg J, Cremer C, Cremer T, Solovei I: Arrangements of macro- and microchromosomes in chicken cells. Chromosome Res 9:569-584 (2001).

Haraguchi T, Shimi T, Koujin T, Hashiguchi N, Hiraoka Y: Spectral imaging fluorescence microscopy. Genes Cells 7:881-887 (2002).

Ishida T: A brief report on Primate Cell Repository (PCRT) in Japan, in Ehara A, Kimura T, Takenaka O, Iwamoto M (eds.): Primatology Today: Proceeding of the XIIIth Congress of the International Primatological Society, pp 655-656 (Elsevier, Amsterdam 1991).

Kimura H, Cook PR: Kinetics of core histones in living human cells: little exchange of $\mathrm{H} 3$ and $\mathrm{H} 4$ and some rapid exchange of H2B. J Cell Biol 153: 1341-1353 (2001).

Kozubek S, Lukasova E, Jirsova P, Koutna I, Kozubek M, Ganova A, Bartova E, Falk M, Pasekova R: 3D Structure of the human genome: order in randomness. Chromosoma 111:321-331 (2002).

Lamond AI, Earnshaw WC: Structure and function in the nucleus. Science 280:547-553 (1998)

Leitch AR: Higher levels of organization in the interphase nucleus of cycling and differentiated cells. Microbiol Mol Biol Rev 64:138-152 (2000). 
Müller S, O’Brien PC, Ferguson-Smith MA, Wienberg $\mathrm{J}$ : A novel source of highly specific chromosome painting probes for human karyotype analysis derived from primate homologues. Hum Genet 101:149-153 (1997).

Parada L, Misteli T: Chromosome positioning in the interphase nucleus. Trends Cell Biol 12:425-432 (2002).

Phair RD, Misteli T: High mobility of proteins in the mammalian cell nucleus. Nature 404:604-609 (2000).

Solovei I, Walter J, Cremer M, Habermann F, Schermelleh L, Cremer T: FISH on three-dimensionally preserved nuclei, in Squire J, Beatty B, Mai S (eds): FISH: A Practical Approach, pp 119-157 (Oxford University Press, Oxford 2002).

Stanyon R, Ardito G, Lamberti L, Bigatti P: The banded karyotypes of Macaca fuscata compared with Cercocebus aterrimus. Folia Primatol 41:137146 (1983).
Stanyon R, Romagno D, Wienberg J, Maurer U: Sequence of DNA replication in Macaca fuscata chromosomes: an outgroup for phylogenetic comparison between man and apes. Genetica 80:45-52 (1990).

Stanyon R, Arnold N, Koehler U, Bigoni F, Wienberg J: Chromosomal painting shows that "marked chromosomes" in lesser apes and Old World monkeys are not homologous and evolved by convergence. Cytogenet Cell Genet 68:74-78 (1995).

Tanabe H, Habermann FA, Solovei I, Cremer M, Cremer T: Non-random radial arrangements of interphase chromosome territories: evolutionary considerations and functional implications. Mutat Res 504:37-45 (2002a).

Tanabe H, Müller S, Neusser M, von Hase J, Calcagno E, Cremer M, Solovei I, Cremer C, Cremer T: Evolutionary conservation of chromosome territory arrangements in cell nuclei from higher primates. Proc Natl Acad Sci USA 99:4424-4429 (2002b).
Telenius H, Carter NP, Bebb CE, Nordenskjold M, Ponder BA, Tunnacliffe A: Degenerate oligonucleotide-primed PCR: general amplification of target DNA by a single degenerate primer. Genomics 13:718-725 (1992)

Walter J, Schermelleh L, Cremer M, Tashiro S, Cremer T: Chromosome order in HeLa cells changes during mitosis and early G1, but is stably maintained during subsequent interphase stages. J Cell Biol 160:685-697 (2003).

Wienberg J, Stanyon R: Comparative chromosome painting of primate genomes. ILAR J 39:77-91 (1998).

Wienberg J, Stanyon R, Jauch A, Cremer T: Homologies in human and Macaca fuscata chromosomes revealed by in situ suppression hybridization with human chromosome specific DNA libraries. Chromosoma 101:265-270 (1992). 